\title{
Percepción del profesorado latinoamericano y español sobre el cambio climático: aproximaciones desde un MOOC de formación docente
}

\author{
Diego Corrochano Fernández, Enzo Rainiero Ferrari Lagos, \\ Santiago Andrés Sánchez, Miguel Ángel Fuertes Prieto, Pablo Herrero Teijón, \\ Anne Marie Ballegeer, Laura Delgado Martín, Camilo Ruiz Méndez \\ Departamento de Didáctica de la Matemática y de las Ciencias Experimentales, \\ Universidad de Salamanca, España.dcf@usal.es, enzoferrari@usal.es, \\ santiandres@usal.es,fuertes@usal.es,pabloherrero@usal.es,amballegeer@usal.es, \\ laura@usal.es,camilo@usal.es \\ ORCID: https://orcid.org/0000-0002-6085-9744, https://orcid.org/0000-0002-4533-021X, \\ https://orcid.org/0000-0002-8088-1607, https://orcid.org/0000-0002-6914-6871, \\ https://orcid.org/0000-0001-7478-1637, https://orcid.org/0000-0001-6296-1868, \\ https://orcid.org/0000-0001-7203-1832, https://orcid.org/0000-0001-9538-5780
}

[Recibido: 15 febrero 2021. Revisado: 19 noviembre 2021. Aceptado: 28 enero 2022]

\begin{abstract}
Resumen: Este estudio se centra en analizar las creencias que tienen profesores de España y Latinoamérica sobre el cambio climático, prestando especial atención a la percepción general que tienen del fenómeno, la concienciación, las preocupaciones y al conocimiento de determinados fundamentos científicos. Para recoger la información, se utilizó un cuestionario de 38 ítems (preguntas y afirmaciones) de respuesta cerrada que respondieron un total de 85 profesores de niveles preuniversitarios durante un curso online de formación docente sobre el cambio climático. Independientemente del lugar de procedencia, España o América Latina, los profesores presentan entre ellos resultados muy similares en los aspectos considerados en el estudio, registrándose diferencias significativas en muy pocos de ellos. Creen que el fenómeno es algo real y provocado por la actividad humana, pero a pesar de reconocer la amenaza para la salud que supone, sostienen que afectará más gravemente a futuras generaciones. Ambos grupos comparten la idea de que la sociedad no está preparada para adoptar medidas de adaptación y mitigación. Además, se ha detectado entre el profesorado varias concepciones alternativas sobre los fundamentos científicos y los conocimientos conceptuales que tienen sobre el problema, que están en concordancia con estudios similares realizados en otros contextos con la población en general. Por último, se discuten algunas implicaciones en la formación docente.
\end{abstract}

Palabras clave: Cambio climático; Educación; profesores; España; Latinoamérica.

Latin American and Spanish teachers' perception of climate change: approaches from a teacher education MOOC

Abstract: This study focuses on analyzing the beliefs that Spanish and Latin American teachers have about climate change, paying special attention to their general perception of the phenomenon, awareness, concerns and knowledge about certain scientific concepts. To collect information, a questionnaire of 38 closed-response items (questions and statements) was used, which was answered by a total of 85 pre-university teachers during an online teacher education course on climate change. Regardless of their place of origin, Spain or Latin America, the teachers presented very similar results in the aspects considered in the study, with very few of them showing significant differences. Teachers believe that the phenomenon is real and caused by human activity, but despite recognizing the threat to health it poses, they perceive it as something that will affect more to future generations. Both groups share the idea that society is not prepared to adopt adaptation and mitigation measures. In addition, 
several misconceptions about the scientific knowledge have been detected, which are in agreement with similar studies carried out in other contexts with general population. Finally, some implications for teacher education are discussed.

Keywords: Climate change; Education; Teachers; Spain; Latin America.

Para citar este artículo: Corrochano Fernández, D., Ferrari-Lagos, E., Andrés-Sánchez, S., Fuertes Prieto, M. A., Herrero-Teijón, P., Ballegeer, A. M., Delgado-Martín, L., \& Ruiz Méndez, C. (2021). Percepción del profesorado latinoamericano y español sobre el cambio climático: aproximaciones desde un MOOC de formación docente. Revista de Educación Ambiental y Sostenibilidad 3(2), 2604. doi: 10.25267/Rev_educ_ambient_sostenibilidad.2021.v3.i2.2604

\section{Introducción}

El cambio climático (CC) constituye uno de los retos más importantes a los que se enfrenta la humanidad en el siglo XXI, amenaza gran parte de la biosfera y prácticamente la totalidad de las actividades humanas (Intergovernmental Panel on Climate Change, IPCC, 2021). Tal es la gravedad del problema, que en un estudio reciente llevado a cabo en 50 países con más de la mitad de la población mundial, el $64 \%$ de los participantes consideró que el CC sigue constituyendo una emergencia global a pesar de la crisis sanitaria de la COVID19 (United Nations Development Programme, UNDP, 2021). Los efectos negativos del calentamiento global sobre los sistemas naturales y humanos, hacen necesario movilizar a la sociedad en general, y a cada individuo en particular, para crear una respuesta coherente y coordinada ante este grave problema. Hay un consenso generalizado entre la comunidad científica de que existe una relación causal entre las actividades humanas y el CC (Cook et al., 2016). Existen un convincente número de pruebas de que el CC es el resultado de la combinación de la variabilidad natural y la influencia humana, en particular los gases de efecto invernadero emitidos por el uso de combustibles fósiles y cambios en el uso de la tierra (IPCC, 2021). Así, las sociedades se encuentran ante la urgencia de transformar radicalmente el modelo energético y el modo de vida de sus ciudadanos. Para ello, surge la necesidad de formar o alfabetizar a las personas de una manera adecuada, para que puedan tomar decisiones, tanto en el presente como en el futuro, de una manera formada e informada.

En el objetivo 13 (Acción por el clima) de la Agenda de la Educación para el Desarrollo Sostenible, se reconoce que para adoptar medidas urgentes para combatir el cambio climático y sus efectos es necesario mejorar la educación, la sensibilización y la capacidad humana e institucional respecto de la mitigación del cambio climático, la adaptación a él, la reducción de sus efectos y la alerta temprana (ONU, 2015). Sin embargo, es evidente que la educación no podrá por sí sola llevar a cabo el gran desafío de hacerse cargo de un cambio tan profundo en nuestras sociedades. A diferencia por ejemplo de la gran crisis ambiental que hubo relacionada con el agujero de ozono, la solución del cambio climático (mitigación y adaptación) es mucho más compleja. El problema del agujero de la capa de ozono comenzó a resolverse de una manera más sencilla porque existían sustitutos, de fácil disposición, para los compuestos que la estaban deteriorando. Sin embargo, la solución del CC requiere de un cambio en el sistema energético y económico mundial, y un cambio radical en el modo de vida de las personas. Esta transición energética no podrá lograrse sin realizar transformaciones en las dimensiones sociales, culturales y éticas 
del problema, y su avance sólo se conseguirá mediante profundos cambios en los procesos educativos vigentes (González-Gaudiano \& Meira-Cartea, 2019).

Desde hace varios años, las opiniones públicas sobre el CC han sido de interés para muchos investigadores y responsables políticos y económicos, con el fin de identificar la percepción humana sobre el fenómeno y poder articular una respuesta más eficiente para resolverlo. En los últimos años hay muestras evidentes de que la población en general está más preocupada ante el problema (UNDP, 2021). Existen numerosos movimientos sociales que presionan para dar una respuesta política al CC (Roser-Renouf et al., 2014). Estos movimientos, como "Fridays For Future", "Teachers for Future" o "Extinction Rebellion", surgidos de la preocupación social y la necesidad de una transformación social, están haciendo una labor muy importante de pedagogía, comunicación y reivindicación para intentar modificar nuestro modo de vida. Sin embargo, como indican Tavares et al., (2020), los movimientos sociales pueden ser eficaces a la hora de concienciar sobre la crisis climática actual, pero no lo son tanto a la hora de impulsar un cambio real en la sociedad para que los individuos se comprometan activamente con las cuestiones relacionadas con el medio ambiente y modifiquen sus hábitos diarios de consumo (pasar del "decir hacer" al "hacer"). De hecho, se ha comprobado que en nuestra sociedad sigue existiendo una inercia social con respecto al clima, probablemente impulsada por los medios de comunicación (Tavares et al., 2020), que limita el desarrollo y la aplicación de medidas eficaces para hacer frente al CC (Plein, 2019). Además de esta inercia, en la sociedad existen una serie de barreras económicas, estructurales y sociales que dificultan que el cambio tenga lugar (Semenza et al., 2008).

Mejorar la educación sobre el fenómeno es fundamental para involucrar a los más jóvenes en el debate público actual y futuro de manera crítica y responsable. La educación del CC debe proporcionar a los alumnos una serie de habilidades y capacidades transversales que enriquezcan sus dominios cognitivos y afectivos (Mochizuk \& Bryan, 2015). Además, debe ser una tarea interdisciplinar que englobe los aspectos científicos, socio-económicos y las posibles soluciones (Stevenson et al., 2017), es decir, debe educar para el clima y para el cambio (González-Gaudiano y Meira-Cartea, 2019). Desde esta perspectiva, las escuelas deben fomentar la participación, el manejo de la información y la toma de decisiones de manera libre y consciente (Varela-Losada et al., 2018). Sin embargo, a pesar de que la educación está reconocida como una herramienta fundamental en la lucha contra el CC, todavía no existe una estrategia clara de cómo implementarla de manera efectiva. La respuesta educativa al CC es muy desigual y existen publicaciones recientes que demuestran un abanico de propuestas que conducen a diferentes niveles de promulgación y asimilación (Drewes et al., 2018).

Es evidente que para mejorar la respuesta educativa en su conjunto con una visión a medio-largo plazo, es necesario que las universidades reorienten la formación del profesorado -de los distintos niveles educativos- hacia la sostenibilidad, y que los docentes tengan una formación suficiente y adecuada para plantear prácticas educativas centradas en el desarrollo de competencias y habilidades, promoviendo entre sus alumnos prácticas sostenibles y responsables con el entorno (Varela-Losada et al. 2018). Además, es necesario concienciar al profesorado sobre el problema, ya que podría proporcionarles más conocimientos sobre el mismo (Dal et al. 2015). Es decir, no se puede lograr una educación eficaz sobre el CC sin centrar esfuerzos en las 
personas que tienen la responsabilidad directa de educar, transmitir información, ideas e innovaciones relacionadas con la sostenibilidad en general y el CC en particular.

Por lo tanto, es importante insistir en el papel protagonista que desempeña el profesorado a la hora de impartir una educación básica sobre el CC. Un primer paso necesario para plantear esta reorientación y convertir a los docentes en verdaderos agentes de cambio, es conocer y comprender los conocimientos e inquietudes que tienen sobre el CC, especialmente aquellas relacionadas con aspectos generales (aceptación social, confianza, preocupaciones, etc.) y con los conocimientos científicos básicos del problema. De hecho, investigaciones previas han demostrado que no solo los alumnos tienen concepciones alternativas sobre muchas cuestiones medioambientales, incluido el CC, sino que también las tienen el profesorado y el profesorado en formación inicial, con el posible efecto multiplicador que ello puede conllevar (Dove, 1996; Papadimitriou, 2004).

Para ayudar a mitigar los efectos negativos del CC desde el ámbito educativo, es necesario conocer mejor las actitudes, comportamientos y percepciones de los diferentes agentes implicados, prestando especial atención al profesorado. Existen varios estudios sobre los conocimientos, las creencias y la percepción general del CC entre el profesorado y el profesorado en formación inicial en diferentes países y contextos educativos, como por ejemplo en Australia (Boon, 2010), Grecia (Dal et al., 2015; Ikonomidis et al., 2012; Papadimitriou, 2004), Turquía (Higde et al., 2017), EEUU (Stevenson et al., 2016) o Canadá (Puk \& Stibbards, 2012). En lo que se refiere al profesorado y al profesorado en formación de habla hispana (latinoamericanos y españoles), existen relativamente pocos estudios centrados en analizar la visión que tienen sobre el fenómeno (Lopera et al., 2021; Morote et al., 2021). En la mayor parte de todos los trabajos citados anteriormente, se identifica que los profesores y los profesores en formación no han recibido una formación adecuada sobre el CC, sus causas, consecuencias y posibles soluciones, lo que a su vez podría reflejarse en una inadecuada educación ambiental en los centros educativos. Por este motivo, PérezRodríguez et al., (2017) pusieron de manifiesto que la formación inicial y continua del profesorado debe favorecer la alfabetización climática para garantizar el desarrollo de capacidades, la participación y la innovación alrededor de esta temática.

Teniendo en cuenta lo expuesto anteriormente, en este trabajo, a partir de datos recogidos en un MOOC (Massive Online Open Course) de formación docente sobre el CC, se presentan algunas creencias sobre el CC que tienen profesores de España y Latinoamérica, prestando especial atención a la percepción general del fenómeno, la concienciación, las preocupaciones y a determinados fundamentos científicos.

\section{Metodología}

\section{Participantes}

Para realizar esta investigación se ha aplicado un cuestionario de opinión a los profesores $(n=85)$ que participaron y se identificaron como tal en la primera edición del MOOC "Concienciación y capacitación en materia de cambio climático para profesores de Primaria y Secundaria" (Herrero et al., 2018), ofertado a través de la plataforma MiriadaX. De los alumnos que iniciaron el curso, se extrajo en primer lugar información de los 90 profesores de Educación Primaria o Secundaria que aceptaron participar de manera voluntaria en el estudio y sus datos 
sociodemográficos se recogen en la Tabla 1. Los participantes tenían una media de edad de 38 años (SD=12,8). El 51\% eran mujeres y el $49 \%$ hombres. De ellos, el 37,8\% eran españoles, el 56,7\% procedía de varios países de Latinoamérica (México, Argentina, Perú, Ecuador, Honduras, República Dominicana, Nicaragua, Costa Rica, Paraguay, Venezuela, Guatemala, Chile y Panamá) y el 5,5\% restante de otros lugares del mundo (Italia, EEUU, Portugal y Congo); estos últimos profesores fueron descartados para los análisis que se presentan en este artículo, por lo que la muestra final analizada es $n=85$. El profesorado español procedía, al menos, de 10 comunidades autónomas diferentes.

Tabla 1. Variables sociodemográficas de la muestra de estudio. Fuente: elaboración propia.

\begin{tabular}{llcl}
\hline Variables & & No $(\mathrm{n}=90)$ & $\%$ \\
\hline Género & Femenino & 46 & 51,1 \\
& Masculino & 44 & 48,9 \\
& $<25$ años & 17 & 18,9 \\
& 25-45 años & 46 & 51,1 \\
País de & $>45$ años & 27 & 30,0 \\
residencia & España & 34 & 37,8 \\
& México & 9 & 10,0 \\
& Argentina & 9 & 10,0 \\
& Perú & 6 & 6,7 \\
& Ecuador & 5 & 5,5 \\
& Otros América & 22 & 24,5 \\
& Latina & 5 & 5,5 \\
& Otros & & \\
\hline
\end{tabular}

\section{Instrumento}

El presente trabajo se ha realizado siguiendo un enfoque cuantitativo de investigación. Para conocer las ideas generales de los profesores sobre el CC, se utilizó un cuestionario adaptado de trabajos publicados previamente en diferentes contextos (Akerlof et al., 2010; Meira-Cartea et al., 2018; Stevenson et al., 2016; Tobler et al., 2012). Está formado por 38 ítems (preguntas y afirmaciones) de respuesta cerrada que se pueden agrupar en tres grandes bloques. El primero consta de veinte afirmaciones que se responden mediante una escala de Likert 1-5 (1: muy poco o muy en desacuerdo y 5: mucho o muy de acuerdo). Además de preguntar sobre aspectos relativos a la percepción general del problema, la preocupación, concienciación o motivación, las preguntas inciden en la variable temporal (generaciones actuales o generaciones futuras), la variable económica (países desarrollados o países en vías de desarrollo), la cercanía al propio individuo (propia familia o propia persona) y el impacto en el medio natural. El segundo bloque, está formado por dos preguntas y consiste en establecer y priorizar las consecuencias del CC que más preocupan a los encuestados. El tercer bloque está formado por dieciséis afirmaciones cuyas alternativas de respuesta se organizan en una escala de sí o no (sí o no se verán afectadas esas afirmaciones por el CC). Están relacionadas con la salud y la enfermedad y el CC -la probabilidad de que el CC afecte a la salud de las personas y de que aumente el riesgo de padecer una serie de enfermedades concretas-, y una serie de acciones cotidianas que contribuyen al CC. 
Con todas estas cuestiones se pretendía evaluar qué percepción, grado de concienciación y preocupación, y conocimientos generales tiene el profesorado sobre el problema. Todos los alumnos, independientemente del país de origen, respondieron exactamente al mismo cuestionario. Esto se hizo de manera voluntaria, a través de un formulario electrónico, sin límite de tiempo establecido, y antes de comenzar el MOOC.

\section{Análisis de datos}

En la primera parte del cuestionario se analizó nuestra hipótesis sobre la existencia de diferencias significativas entre los profesores de España y de Latinoamérica en las acciones, cambios de estilo de vida e importancia de luchar contra el CC. Para responder esto, empezamos aplicando el test de Kolmogorov-Smirnov para determinar la normalidad de las variables. Los resultados del $p$-valor fueron $<0,05$, lo que sugiere que los datos no presentan distribuciones normales, por lo que se trabajó con estadística no paramétrica. Para el contraste de hipótesis, se aplicó el test de U de Mann-Whitney, que compara las distribuciones de dos grupos independientes cuando los datos no presentan normalidad. En todos los casos, el cálculo de las diferencias fue acompañado con el tamaño del efecto, basado en el estadístico de $d$ de Cohen (Cohen, 2013). Por lo tanto, las diferencias entre ambos grupos no sólo se basaron en los criterios estadísticos proporcionados por el contraste de hipótesis, sino también en la cantidad de efecto existente en el contraste en particular. Estas diferencias fueron consideradas como pequeñas $(d=0,20)$, moderadas $(d=0,50)$ o grandes $(d=0,80)$.

Para el segundo y tercer bloque del cuestionario continuamos con la comprobación de nuestra hipótesis sobre la existencia de diferencias por región en los ítems de variables categóricas (aumento del nivel del mar e inundaciones, enfermedades que pueden causar alergias e infecciones que pueden causar diarreas extremas). Para ello aplicamos un análisis de chi cuadrado o test exacto de Fisher (si existe una categoría menor a 5), para determinar la existencia o no de relación entre ambas variables. El Chi-cuadrado y el test exacto de Fisher son estadísticos de significación y deben ir acompañados de una estadística que determine la fuerza de asociación entre las dos variables. Cuando ambas variables son dicotómicas, el Odds Ratio (OR), es un excelente estadístico para determinar el tamaño de la asociación. Puede tomar valores de 0 a infinito positivo. Cuando este es mayor que 1 , se interpreta como la cantidad de veces que algo pueda suceder sobre que no pueda suceder. Un OR de 1,5 a 2,4, indica una asociación débil, de 2,5 a 3,9, asociación moderada, y mayores a 4,0, fuertes (Rosenthal, 2012). También es posible utilizar el coeficiente phi $(\varphi)$, para determinar la fuerza de asociación. Un valor de $\varphi=0,1$ es considerado un efecto pequeño, 0,3 un efecto medio, y 0,5 un efecto grande. El nivel de significación usado en todos los contrastes se analizó bajo el $5 \%$.

\section{Contexto de la investigación: MOOC de formación docente sobre la ciencia básica del cambio climático}

El curso "Concienciación y capacitación en materia de cambio climático para profesores de Primaria y Secundaria" fue desarrollado y realizado por un equipo interdisciplinar de profesores de la la Universidad de Salamanca (especialistas en diversas ramas del conocimiento científico y la educación) y está alojado en la plataforma de habla hispana MiriadaX. El MOOC tiene una duración total certificada de 30 horas y sus contenidos se organizan siguiendo las pautas generales que marca 
la plataforma de alojamiento, es decir, seis módulos desarrollados en aproximadamente seis semanas de duración. La estructura del curso está basada en torno a cuatro grandes preguntas: ¿Qué es el CC? ¿Cuáles son sus causas? ¿Cuáles son sus consecuencias? ¿Qué podemos hacer? Las tres primeras preguntas son respondidas a través de explicaciones científicas. La última está relacionada con los conceptos de adaptación y mitigación, y cómo la educación puede ayudar a crear conciencia ciudadana, promover el conocimiento y ayudar a construir capacidades y estrategias para enfrentarse a este problema global. En el curso se incluye una colección de vídeos de gran calidad, así como recursos multimedia, adaptados al currículo oficial de la LOMCE y también siguiendo las directrices del IPCC (Intergovernmental Panel on Climate Change).

Los datos del presente estudio se recogieron en la primera edición del curso, que se llevó a cabo durante los meses de julio y agosto de 2018, tras tres semanas de preinscripción. A pesar de que el curso se realizó durante el periodo vacacional de verano y del escaso tiempo de preinscripción que hubo, la primera edición tuvo un éxito considerable, con 1215 inscritos (40\% eran de nacionalidad española y el resto procedían de América Latina), de los cuales 800 lo iniciaron y 101 lo finalizaron.

\section{Resultados}

Los resultados obtenidos sobre la percepción general del fenómeno que tienen los profesores se muestran en la Figura 1. Como puede observarse, cerca del $40 \%$ de los participantes considera que su grado de formación sobre el CC es bueno o muy bueno, y únicamente un 15,3\% y $2,4 \%$ señala que es poco o muy poco, respectivamente. Una amplia mayoría (95,3\%) afirma que es un problema que está ocurriendo realmente y que es causado por actividades humanas (88,2\%). En contraste, el 14,1\% y el 11,8\% están de acuerdo o muy de acuerdo en que el CC se debe a causas naturales. Además, un elevado porcentaje muestra confianza o mucha confianza en lo que la ciencia dice sobre el mismo $(78,9 \%)$, reconoce estar preocupado o muy preocupado ante el problema $(88,2 \%)$ y entiende $(81,2 \%)$ que sus acciones individuales son parte de la solución y estaría dispuesto a cambiar su estilo y nivel de vida para paliar sus efectos. A pesar de todo esto, más de la mitad de los encuestados piensa que la sociedad no está preparada para adoptar medidas de adaptación y mitigación frente al CC, un $23,5 \%$ se declara neutral y solo un $5,9 \%$ y un $16,5 \%$ considera que sí está preparada. Cuando se pregunta al profesorado sobre la naturaleza del problema en cuestión, una amplia mayoría entiende que es un problema global, de índole social, educativo, político, económico y medioambiental, pero únicamente un 47,1\% reconoce el papel de la ciencia en el fenómeno, bien sea para diagnosticarlo o para solucionarlo. Respecto a la pregunta de "¿Cómo de importante crees que es el problema del CC para...", aproximadamente un 55\% y un 22\% reconoce que el problema afecta bastante o mucho tanto a su persona, como a sus familias, afectando por igual a las personas de los países desarrollados o en vías de desarrollo. En este apartado, llama la atención cómo los participantes otorgan mucho más valor a la afirmación de que el CC afectará a futuras generaciones más que a las presentes, reconociendo un 80,0\% que afectará bastante y 11,8\% que lo hará mucho. 


\section{Percepción general del problema}

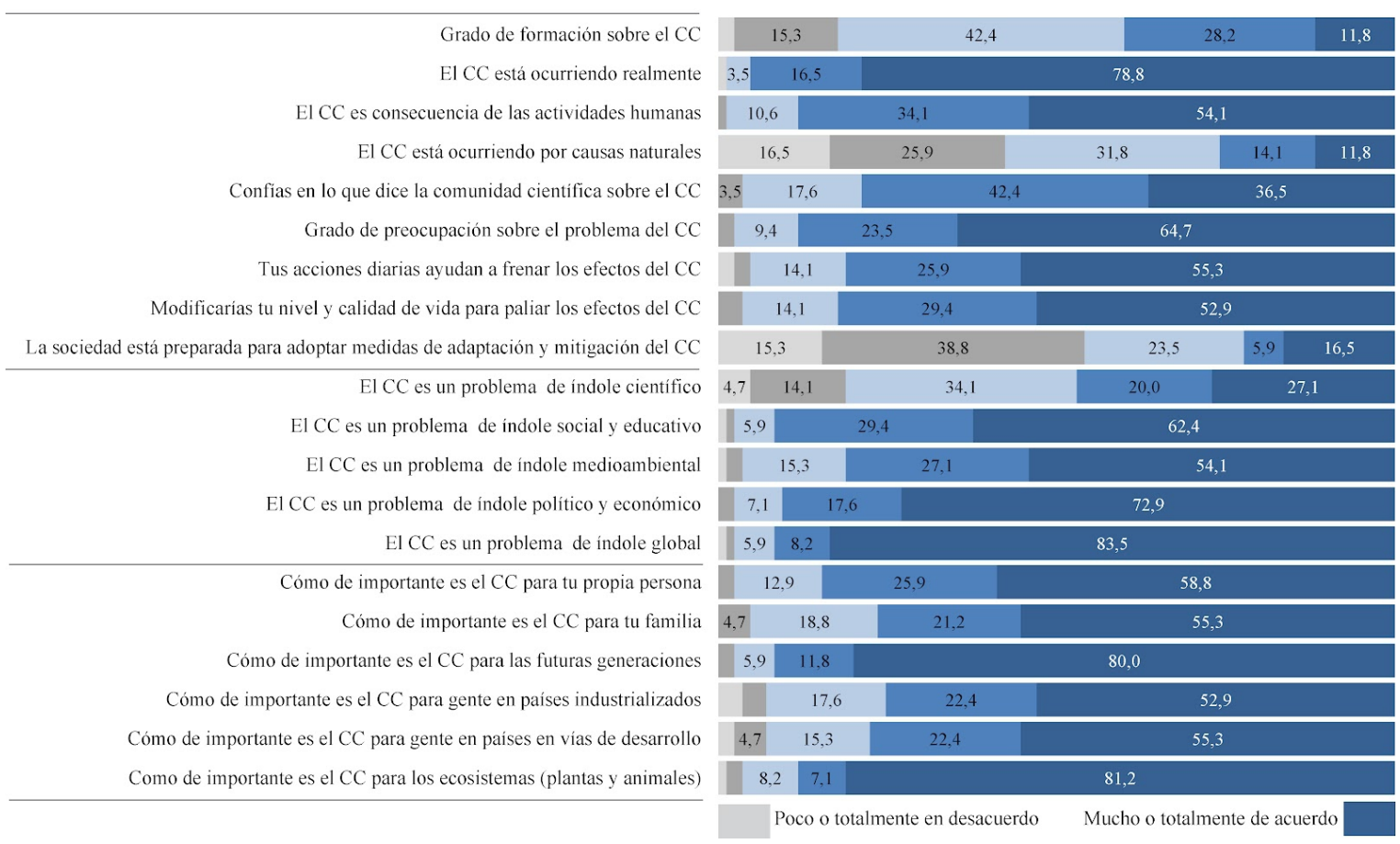

Figura 1. Respuestas (\%) sobre la percepción general del fenómeno. Fuente: elaboración propia.

En lo referente a las consecuencias del CC que más preocupan al profesorado (Fig. 2), una gran mayoría reconoce que el aumento de las temperaturas es lo que más les preocupa, tanto en primera como en segunda opción $(41,2 \%$ y $30,6 \%$, respectivamente). La destrucción de ecosistemas, el aumento del nivel del mar y la escasez de agua potable son las otras tres consecuencias que más preocupan en primer lugar al profesorado. En lo relativo a la segunda máxima preocupación, además del ya mencionado aumento de las temperaturas, los profesores resaltan la destrucción de ecosistemas, inestabilidades geopolíticas y migraciones derivadas y una posible disminución de la calidad de vida.

\section{¿Qué consecuencias del cambio climático te preocupan más?}

Aumento de las temperaturas

Aumento de los eventos meteorológicos extremos

Aumento del nivel del mar e inundación de las zonas costeras

Destrucción de ecosistemas

Disminución del nivel de vida

Efectos sobre la salud

Escasez de agua potable

Incendios forestales

Sequías prolongadas

Inestabilidades geopoliticas y

migraciones derivadas

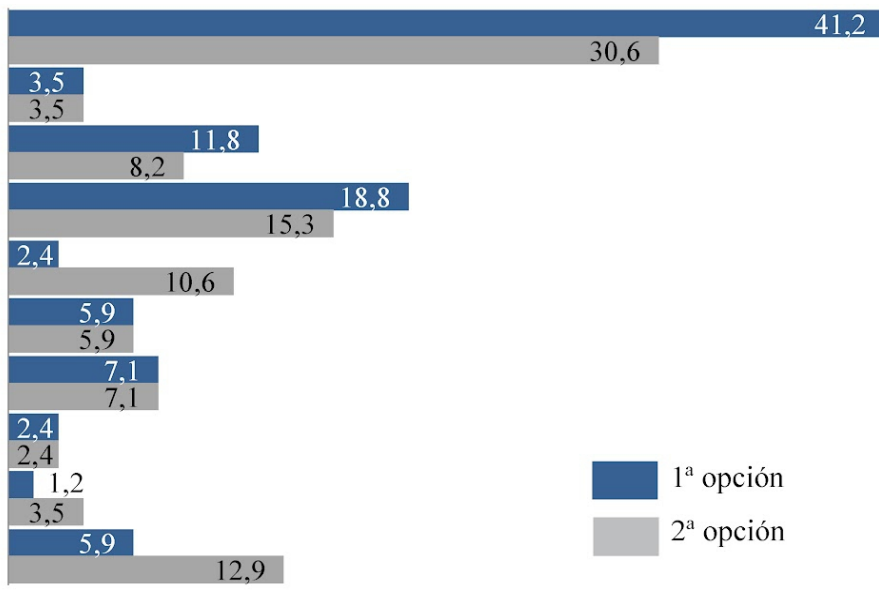

Figura 2. Consecuencias del CC que más preocupan al profesorado encuestado. Fuente: elaboración propia. 
En la Figura 3 se muestran por un lado, los resultados obtenidos respecto a los conocimientos sobre la relación del CC y la salud, y por otro, sobre la relación que tienen algunas acciones cotidianas con el fenómeno. En términos generales, los profesores son conscientes de que el CC puede causar problemas de salud respiratorios, relacionados con el calor, alergias y enfermedades infecciosas. Únicamente cerca del 59\% reconoció que las afecciones cardiovasculares pueden verse afectadas por el fenómeno y un amplio porcentaje de participantes $(91,8 \%)$ afirmó que el CC también podía ocasionar cáncer de piel.

Respecto a las acciones que contribuyen a aumentar el CC, llama la atención que 9 de cada 10 profesores considera que la destrucción de la capa de ozono juega un papel importante. A su vez, el 74,1\% del profesorado afirma que la energía nuclear contribuye a aumentar el problema, el $45,9 \%$ lo hace sobre las redes de telefonía móvil y WIFI, y el 70,6\% de los encuestados reconoce el papel de la agricultura y la ganadería en las emisiones de gases invernadero.

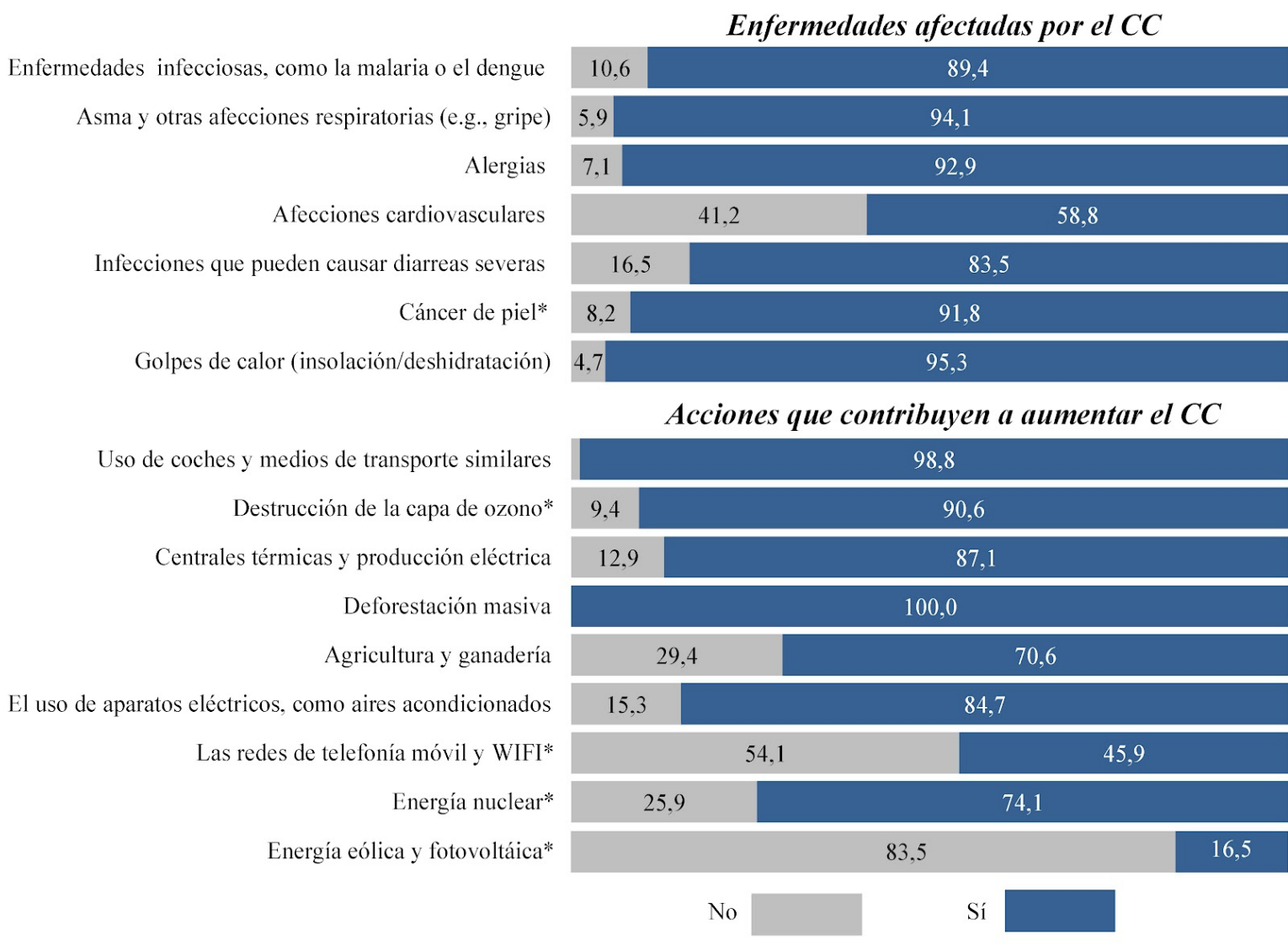

Figura 3. Percepción de los docentes sobre la probabilidad de que algunas enfermedades se vean afectadas por el CC y sobre la contribución de determinadas acciones a aumentar el CC. * Afirmaciones incorrectas de acuerdo al consenso científico actual. Fuente: elaboración propia.

\section{Análisis comparativo entre el profesorado latinoamericano y español}

En las tablas 2, 3 y 4 se muestra un análisis comparativo entre los profesores participantes de América Latina $(n=51)$ y España $(n=34)$ y los aspectos considerados en el trabajo. 
Tabla 2. Comparativa de la percepción general del problema entre profesores latinoamericanos y españoles. Fuente: elaboración propia.

\begin{tabular}{|c|c|c|c|c|c|c|c|c|c|}
\hline \multirow{3}{*}{ Item } & \multicolumn{3}{|c|}{ América Latina } & \multirow{3}{*}{$M$} & \multirow{3}{*}{$\begin{array}{l}\text { España } \\
M d n\end{array}$} & \multirow{3}{*}{$S D$} & \multicolumn{2}{|c|}{$\begin{array}{l}\text { Contraste de } \\
\text { Hipótesis }\end{array}$} & \multirow[b]{2}{*}{$\mathrm{U} p$} \\
\hline & \multirow[t]{2}{*}{$M$} & \multirow[t]{2}{*}{$M d n$} & \multirow[t]{2}{*}{$S D$} & & & & $Z$ & $\mathrm{M}$ & \\
\hline & & & & & & & & $p$ & $d$ \\
\hline $\begin{array}{l}\text { Tus acciones diarias ayudan a } \\
\text { frenar los efectos del CC }\end{array}$ & 4,45 & 5,00 & 1,01 & 4,06 & 4,00 & 0,85 & $-2,72$ & ,007 & ,418 \\
\hline $\begin{array}{l}\text { Modificarías tu nivel y calidad } \\
\text { de vida para paliar los efectos } \\
\text { del CC }\end{array}$ & 4,49 & 5,00 & 0,83 & 4,06 & 4,00 & 0,81 & $-2,77$ & ,006 & ,522 \\
\hline $\begin{array}{l}\text { Cómo de importante es el CC } \\
\text { para tu propia persona }\end{array}$ & 4,59 & 5,00 & 0,70 & 4,15 & 4,00 & 0,89 & $-2,48$ & ,013 &, 549 \\
\hline
\end{tabular}

M: media, Mdn: mediana, $S D$ : desviación estándar, Z: valor de Z, MWU p: p valor, d: tamaño del efecto.

En lo relativo a la comparativa de la percepción general del problema entre profesores latinoamericanos y españoles (Tabla 2), se observan diferencias significativas en las afirmaciones: "Tus acciones diarias ayudan a frenar los efectos del cambio climático", "Modificación del nivel de vida para paliar los efectos del CC" y "El CC es importante para tu propia persona" $(Z=2,72, p<0,05),(Z=-2,77, p<0,05)$ y $(Z=-2.48, p<0,05)$, siendo los profesores latinoamericanos los que alcanzan un nivel de percepción superior en ambas variables (MdnLat $=5$; MdnEsp= 4; respectivamente). El valor del tamaño del efecto explica que las diferencias son moderadas o cercanas a esta.

De todas las consecuencias del CC que preocupa a los profesores, el aumento del nivel mar y las inundaciones asociadas, es la única que presenta una asociación estadísticamente significativa con la región $\left(\chi^{2}(1, N=85)=7,025, \mathrm{p}<0,001\right)$. Se encontró que esta relación es moderada y directamente proporcional $(\varphi=0,289, \mathrm{p}<$ 0,001 ), lo que explica que el $49 \%$ de los profesores latinoamericanos (Tabla 3) se identifican 4 veces más preocupados por el aumento del nivel mar e inundaciones producto del CC, frente a los docentes españoles (odds ratio $=3,7$ ).

Tabla 3. Comparativa de las preocupaciones estadísticamente significativas sobre el cambio climático entre profesorado latinoamericano y español (\%). Fuente: elaboración propia.

\begin{tabular}{lccccccc}
\hline \multicolumn{3}{c}{ Aumento del nivel del mar e inundaciones } & \multicolumn{2}{c}{ Total } \\
\hline Región & \multicolumn{2}{c}{ Sí } & \multicolumn{2}{c}{ No } & & $\mathrm{n}$ & $\%$ \\
\hline Latinoamérica & 25 & 49,0 & 26 & 51,0 & 51 & 100 \\
España & 7 & 20,6 & 27 & 79,4 & 34 & 100 \\
\hline Total & 32 & 37,6 & 53 & 62,3 & 85 & 100 \\
\hline
\end{tabular}

En lo relativo al bloque de preguntas relacionadas con los conocimientos, el profesorado latinoamericano muestra una puntuación media sensiblemente inferior al profesorado español. De todas las afecciones a la salud, las únicas que presentan una asociación estadísticamente significativa con la región (España y Latinoamérica) son las que pueden causar alergia (valor $p$ asociado al test exacto de Fisher $=0,041$ ) y 
las infecciones que pueden causar diarreas severas $\left(\chi^{2}(1, N=85)=4,119, p=0,042\right)$. En cuanto a la primera, se encontró que dicha relación es baja y directamente proporcional $(\varphi=0,225, \mathrm{p}=0,038)$, lo que explica que un $12 \%$ más de profesores españoles que de docentes latinoamericanos piensan que enfermedades que causan alergias empeorarán con el CC (Tabla 4). En cuanto a la segunda, se encontró que dicha relación también es baja y directamente proporcional $(\varphi=0,225, p=0,042)$, lo que explica que el 90,2 \% de los profesores latinoamericanos (Tabla 4) se identifican 3 veces más preocupados a que las infecciones que producen diarreas severas aumenten con el CC, frente a los docentes españoles (odds ratio = 3,3).

Tabla 4. Comparativa de conocimientos (enfermedades afectadas y acciones que afectan) que muestran diferencias significativas sobre el cambio climático entre profesores latinoamericanos y españoles. Fuente: elaboración propia.

\begin{tabular}{|c|c|c|c|c|c|c|}
\hline \multirow[b]{3}{*}{ Región } & \multicolumn{4}{|c|}{$\begin{array}{l}\text { Enfermedades que pueden causar } \\
\text { alergias }\end{array}$} & & \\
\hline & \multicolumn{2}{|c|}{ Sí } & \multicolumn{2}{|c|}{ No } & \multicolumn{2}{|c|}{ Total } \\
\hline & $\mathrm{n}$ & $\%$ & $\mathrm{n}$ & $\%$ & $\mathrm{n}$ & $\%$ \\
\hline Latinoamerica & 45 & 88,2 & 6 & 11,8 & 51 & 100 \\
\hline España & 34 & 100,0 & 0 & 0,0 & 34 & 100 \\
\hline \multirow[t]{3}{*}{ Total } & 79 & 92,9 & 6 & 7,1 & 85 & 100 \\
\hline & \multicolumn{4}{|c|}{$\begin{array}{l}\text { Enfermedades que pueden causar } \\
\text { diarreas }\end{array}$} & & \\
\hline & \multicolumn{2}{|c|}{ Sí } & \multicolumn{2}{|c|}{ No } & \multicolumn{2}{|c|}{ Total } \\
\hline Región & $\mathrm{n}$ & $\%$ & $\mathrm{n}$ & $\%$ & $\mathrm{n}$ & $\%$ \\
\hline Latinoamerica & 46 & 90,2 & 5 & 9,8 & 51 & 100 \\
\hline España & 25 & 73,5 & 9 & 26,5 & 34 & 100 \\
\hline Total & 71 & 83,5 & 53 & 16,5 & 85 & 100 \\
\hline
\end{tabular}

\section{Discusión}

Los resultados de este trabajo muestran que los profesores españoles y latinoamericanos tienen unas ideas sobre el CC muy similares a las que muestra la población en general en diferentes contextos de todo el mundo. Además, independientemente de su lugar de origen, estos profesores presentan entre ellos resultados muy similares en las percepciones, la concienciación, las preocupaciones y los conocimientos básicos considerados en el estudio, registrándose diferencias significativas en muy pocos de los aspectos analizados.

La mayoría de los docentes que participaron en la encuesta reconocieron tener unos conocimientos moderados sobre el CC, algo que concuerda con los resultados obtenidos en alumnos universitarios españoles de diferentes ramas del conocimiento (Meira-Cartea et al., 2018). Los datos obtenidos indican también que la negación del CC es prácticamente inexistente entre el profesorado, datos que están en consonancia por ejemplo con los presentados por la población en general en España, en los que el 90,1\% pensaba que "el cambio climático está ocurriendo" (Meira-Cartea et al., 2013). Una amplia mayoría de participantes $(88,2 \%)$ reconoció que el CC es causado por las actividades humanas, coincidiendo con lo que afirman casi todos (>95\%) los 
científicos del clima (Cook et al., 2016) y corroborando la confianza que depositaron los encuestados con lo que dice la comunidad científica $(78,9 \%)$. Los resultados vuelven a asemejarse, aunque son ligeramente superiores en la muestra estudiada, a los obtenidos en la población en general en España, en los que 64,3\% creía que el CC es provocado "principalmente" o "exclusivamente" por causas humanas, y menos de uno de cada diez $(8,4 \%)$ creía que se debe "principalmente" o "exclusivamente" a causas naturales (Meira-Cartea et al., 2013). Otro aspecto que llama la atención de los resultados obtenidos, es que tanto los profesores latinoamericanos como los españoles muestran un gran pesimismo a la hora de plantear si la sociedad está preparada para adoptar medidas de adaptación y mitigación del CC. Esto es preocupante y es necesario tenerlo en cuenta en la formación del profesorado, ya que transmitir una visión optimista y positiva de la solución del problema es algo fundamental para movilizar a la población en políticas de adaptación y mitigación (Ojala, 2012; 2015).

Respecto a la capacidad individual que tenemos las personas para mitigar los efectos del CC, los profesores latinoamericanos se muestran más predispuestos y optimistas que los docentes españoles. Además, los resultados ponen de manifiesto que los profesores latinoamericanos están más preocupados con algunas de las consecuencias producidas por el CC, como el aumento del nivel del mar y las inundaciones. Ferrari et al., (2019), en un estudio llevado a cabo con estudiantes de un MOOC acerca de la ciencia básica del CC, encontraron que los participantes latinoamericanos mostraban una mayor representación social de este problema que los españoles antes de realizar esta formación. Estos autores sugieren que estas diferencias se pueden deber a las diferencias existentes en el currículum oficial de las etapas obligatorias entre los países.

Respecto a las preguntas de “¿Cómo de importante crees que es el problema del CC para...", llama la atención cómo los participantes otorgan mucho más valor a la cuestión de que el CC afectará a futuras generaciones, que a aquella en la que se asegura que afecta a sus propias personas o familias, tanto en países desarrollados como aquellos en vías de desarrollo. Esto sugiere que a pesar de que los profesores contemplan el fenómeno como algo importante en su vida cotidiana, creen que el problema afectará más a las personas en un futuro más o menos lejano. En este sentido, un gran número de trabajos publicados en el campo de la salud y la comunicación de riesgos señala que la sensación personal de riesgo de un individuo es el detonante motivador más poderoso del cambio de comportamiento (Witte \& Allen, 2000). La implicación del público en cuestiones relacionadas con el CC es una tarea especialmente difícil porque a menudo los impactos se perciben como inciertos, en un futuro lejano y sin relevancia personal (Leiserowitz, 2006; Lorenzoni et al,, 2007). Surge por tanto la necesidad de modificar los mensajes de comunicación del fenómeno y de transmitir al profesorado la necesidad de una respuesta urgente desde su ámbito profesional, sin fomentar una comunicación persuasiva (Fischhoff, 2007) o amenazante (Tavares et al., 2020). Todo esto se puede lograr por ejemplo mediante el planteamiento de problemas locales que acerquen el fenómeno a los individuos para que lo perciban como algo cercano y personal (Scannell \& Gifford, 2013).

En lo relativo al bloque de preguntas relacionadas con los conocimientos, el profesorado latinoamericano muestra una puntuación media sensiblemente inferior, no significativa, al profesorado español. Únicamente se han observado diferencias 
estadísticamente significativas en dos de los dieciséis aspectos considerados. Ambos colectivos expresan en gran número su creencia de que el CC puede causar problemas de salud, al igual que ocurre en estudios previos llevados a cabo en otros contextos, como en Canadá y Malta (Akerlof et al., 2010) o la población española en general, donde el $65,7 \%$ considera que es bastante o muy probable que su salud se vea afectada en algún momento por el CC (Heras et al., 2017). En concreto, en el presente trabajo el profesorado indicó que los problemas respiratorios, aquellos relacionados con el calor, alergias o enfermedades infecciosas, son los que más probabilidades tienen de verse afectados. El profesorado entrevistado también presentó ciertas dificultades a la hora de relacionar el CC y el calentamiento global con las afecciones cardiovasculares. El 54,9\% de los latinoamericanos y el 32,4\% de los españoles respondieron que el CC no afecta a estos problemas de salud. Sin embargo, en los últimos años se ha demostrado un aumento de la tasa de mortalidad relacionada con problemas cardiovasculares asociadas a aumentos de temperatura provocados por olas de calor (Chen et al., 2019; Peters \& Schneider, 2020). Es posible que los futuros esfuerzos educativos en la formación inicial del profesorado tengan que considerar aumentar el conocimiento de estos riesgos específicos y garantizar que el público sea consciente de sus peligros, sus síntomas y las medidas preventivas.

En lo referente a las acciones que contribuyen a aumentar los efectos del CC, es destacable que el 16,5\% del profesorado considera que la energía eólica y fotovoltaica es una de las principales causas del CC, el 45,9 \% la telefonía móvil y las redes WIFI, el $74,1 \%$ la energía nuclear y el 90,6\% considera que está originado por el agujero de la capa de ozono. Estos porcentajes son mucho más elevados que los registrados en trabajos previos en diferentes contextos. Por ejemplo, en el estudio llevado a cabo por Tobler et al., (2012) con población suiza, únicamente el 38\% de los encuestados atribuyó erróneamente el origen del CC a la destrucción de la capa de ozono, y solo el $17 \%$ señaló que la producción eléctrica en las centrales nucleares contribuye a la emisión a la atmósfera de gases invernadero y por lo tanto agrava el problema.

Uno de los mayores desafíos para la adaptación y la mitigación del CC es la presencia de concepciones alternativas, ideas que son contradictorias con los conocimientos científicos vigentes y que se asumen como correctos (Carrascosa, 2005; Gómez-Zwiep 2008). Esto es especialmente preocupante porque la falta de conocimiento podría contribuir a un sentimiento de incertidumbre sobre el CC, que en última instancia podría dar lugar a un escepticismo sobre la realidad del mismo, la influencia humana y la necesidad de actuar (Lorenzoni et al., 2007). Las concepciones muchas veces están ampliamente distribuidas en la sociedad. Generalmente se adquieren antes de empezar la instrucción formal y si no se trabajan correctamente se pueden convertir en permanentes (Burgoon et al., 2011). Este estudio pone de manifiesto que muchas de las concepciones alternativas previamente registradas en la literatura en otros contextos y en la población en general, están también ampliamente distribuidas entre los profesores encuestados. Por ejemplo, la creencia de que el CC está motivado por el agujero de ozono, la comparten alumnos de secundaria, (e.g., García-Rodeja \& Lima de Oliveira, 2012), alumnos universitarios de diferentes ramas del conocimiento (e.g., Meira-Cartea et al., 2018) o estudiantes de profesorado en formación inicial (e.g., Khalid, 2001). Nuestros resultados confirmaron la idea errónea de que el aumento de la radiación UV y el riesgo de cáncer de piel son una consecuencia del CC, en la que probablemente influye la confusión con la destrucción de la capa de ozono. En 
concreto, en este estudio la mayor parte de los profesores (90\%) considera que el cáncer de piel es una de las enfermedades que se incrementarán con los efectos del CC. Esta idea aparece en otros estudios, aunque en menor proporción, como el llevado a cabo en la población española en general (Heras et al., 2017) o los estudios realizados en Canadá y en Malta (Akerlof et al., 2010) o Suiza (Tobler et al., 2012).

En este sentido, los resultados obtenidos parecen señalar la necesidad de mejorar el proceso de enseñanza y aprendizaje de los contenidos relacionados con el fundamento científico del fenómeno en la formación del profesorado, y más teniendo en cuenta el efecto multiplicador que estas concepciones pueden tener entre el alumnado. También sugieren la necesidad de replantearse la formación inicial y permanente del profesorado, propiciando el análisis de sus propias concepciones alternativas y de las posibles consecuencias que esto podría producir sobre su práctica educativa, así como revisando las concepciones alternativas que sus potenciales alumnos suelen tener sobre el fenómeno.

Como se indicó anteriormente, es deseable que los docentes tengan una sólida formación científica y pedagógica para plantear prácticas educativas centradas en el desarrollo de competencias y habilidades, promoviendo entre sus alumnos prácticas activas que fomenten el pensamiento crítico y la resolución de problemas, y que sean sostenibles y responsables con el entorno (Varela-Losada et al., 2018). Desde hace mucho tiempo se considera a la educación científica una herramienta imprescindible para afrontar con éxito los grandes desafíos mundiales, porque puede influir en los hábitos de la sociedad y en las relaciones sociales, culturales y económicas (Sharma, 2012). Es decir, desde el ámbito educativo no solo es aconsejable que se aporten competencias teóricas, sino que también se debería aportar a los ciudadanos una serie de instrumentos y mecanismos que les permitan identificar aquellos aspectos relacionados con la problemática ambiental y aportar posibles soluciones.

Algunos ejemplos de estas prácticas pueden ser las iniciativas educativas desarrolladas por el colectivo de "Teachers For Future", como "Recreos Residuos cero", efectivo para despertar una conciencia de consumo responsable, reducir los plásticos en los almuerzos de los centros y fomentar una alimentación saludable, o "Colecaminos", una iniciativa que se centra en la importancia del transporte sostenible a los centros escolares para reducir la emisión de gases de efecto invernadero y mejorar nuestra salud al luchar contra el sedentarismo. Por lo tanto, la educación del CC debería centrarse en el tipo de aprendizaje, fomentar el pensamiento crítico y creativo, y el desarrollo de capacidades que formará a los jóvenes con la información que necesitan para que puedan tomar las decisiones adecuadas para responder al CC (Stevenson et al., 2017). Pero en un contexto educativo como ese, en el que el proceso de enseñanza y aprendizaje se centra en el alumnado, el docente tiene que tener un profundo conocimiento del contenido para poder realizar ejemplificaciones y explicaciones en diferentes contextos, plantear proyectos funcionales y establecer relaciones adecuadas de los conceptos clave a abordar. Si bien en una clase expositiva de corte más tradicional, en la que el docente puede confiar y seguir el libro de texto, no es algo tan imprescindible, siguiendo una metodología más activa sí lo es, ya que por ejemplo durante la realización de un experimento por indagación guiada o durante una discusión, el profesor tiene que ser capaz de responder a diferentes preguntas, aclarar conceptos y generar situaciones de conflicto cognitivo entre sus alumnos. Es decir, como indicó Kikas (2004), podría 
decirse que las ideas erróneas de los profesores actúan como obstáculos, especialmente al utilizar métodos de enseñanza activos y centrados en el estudiante.

\section{Consideraciones finales}

Este estudio señala que el profesorado español y latinoamericano se alinea mayoritariamente con las opiniones de la comunidad científica que consideran el CC como un fenómeno real, provocado por la actividad humana y que es una amenaza para la salud de las personas. Los profesores piensan que esta amenaza se agravará en un futuro más o menos cercano y piensan que la sociedad no está preparada para adoptar medidas de adaptación y mitigación. Independientemente del lugar de procedencia, España o América Latina, los profesores presentan entre ellos resultados muy similares en las percepciones, la concienciación, las preocupaciones y los conocimientos básicos considerados en el estudio, registrándose diferencias significativas en muy pocos de ellos. Únicamente destaca que los profesores latinoamericanos se muestran más predispuestos y optimistas que los docentes españoles para mitigar los efectos del CC, y que están más preocupados con algunas de las consecuencias producidas por el CC, como el aumento del nivel del mar y las inundaciones. Los resultados también indican que los conocimientos conceptuales que tienen los profesores sobre el problema están en concordancia con otros estudios similares realizados en otros contextos. Sin embargo, en muchos casos, estos porcentajes son más elevados. Por último, se han registrado varias concepciones alternativas relativas a los fundamentos científicos del fenómeno, como por ejemplo, la idea de que el CC está ocasionado por la destrucción de la capa de ozono y que puede ocasionar cáncer de piel.

Este trabajo presenta varias limitaciones que tienen que ser consideradas. La primera de ellas es que el profesorado participante probablemente sea un colectivo concienciado y preocupado por el problema, al haberse inscrito en un curso de formación docente sobre el CC, algo que podría producir un sesgo en los resultados obtenidos. El segundo problema potencial es que la muestra, a pesar de contar con una amplia distribución geográfica, debido al procedimiento de muestreo utilizado y al tamaño muestral tan reducido, quizás no sea representativa de la amplia población de profesorado español y latinoaméricano. En este sentido, sería aconsejable realizar futuras investigaciones ampliando la muestra de estudio y caracterizando las especialidades y características del profesorado.

\section{Agradecimientos}

Los autores agradecen los comentarios realizados por los dos revisores anónimos, que han contribuido a mejorar notablemente el manuscrito inicial. Este trabajo ha sido financiado mediante el proyecto I+d+i PID2020-114358RB-I00 "Educación para el Cambio Climático y la Sostenibilidad, un estudio longitudinal de aprendizaje intergeneracional", financiado por la Agencia Estatal de Investigación y Ministerio de Ciencia e Innovación (España).

\section{Referencias bibliográficas}

Akerlof, K., DeBono, R., Berry, P., Leiserowitz, A., Roser-Renouf, C., Clarke, K. L., Rogaeva, A., Nisbet, M., Weathers, M., \& Maibach, E. W. (2010). Public perceptions of climate change as a human health risk: surveys of the United States, Canada and Malta. International journal of environmental research and public health, 7(6), 2559-2606. http://dx.doi.org/10.3390/ijerph7062559 
Boon, H. J. (2010). Climate change? Who knows? A comparison of secondary students and pre-service teachers. Australian Journal of Teacher Education, 35, 104-120. http://dx.doi.org/10.14221/ajte.2010v35n1.9

Burgoon, J. N., Heddle, M. L. \& Duran, E. (2011). Re-examining the similarities between teacher and student conceptions about physical science. Journal of Science Teacher Education, 22(2), 101-114. https://doi.org/10.1007/s10972010-9196-x

Carrascosa, J. (2005). El problema de las concepciones alternativas en la actualidad (parte II). El cambio de concepciones alternativas. Revista Eureka sobre Enseñanza y Divulgación de las Ciencias, 2(3), 388-402. http://dx.doi.org/10.25267/Rev_Eureka_ensen_divulg_cienc.2005.v2.i3.07

Chen, K., Breitner, S., Wolf, K., Hampel, R., Meisinger, C., Heier, M., von Scheidt, W., Kuch, B., Peters, A., \& Schneider, A., for the KORA Study Group. (2019). Temporal variations in the triggering of myocardial infarction by air temperature in Augsburg, Germany, 1987-2014. European heart journal, 40(20), 1600-1608. https://doi.org/10.1093/eurheartj/ehz116

Cook, J., Oreskes, N., Doran, P. T., Anderegg, W. R., Verheggen, B., Maibach, E. W., ... \& Rice, K. (2016). Consensus on consensus: a synthesis of consensus estimates on human-caused global warming. Environmental Research Letters, 11(4), 048002. https://iopscience.iop.org/article/10.1088/1748-9326/11/4/048002/pdf

Cohen, J. (2013). Statistical power analysis for the behavioral sciences. Academic Press.

Dal, B., Ozturk, N., Alper, U., Sonmez, D., \& Cokelez, A. (2015). An Analysis of the Teachers' Climate Change Awareness. Athens Journal of Education, 2(2), 111122. https://www.athensjournals.gr/education/2015-2-2-2-Dal.pdf

Drewes, A., Henderson, J., \& Mouza, C. (2018). Professional development design considerations in climate change education: teacher enactment and student learning. International Journal of Science Education, 40(1), 67-89. https://doi.org/10.1080/09500693.2017.1397798

Dove, J. (1996). Student teacher understanding of the greenhouse effect, ozone layer depletion and acid rain. Environmental education research, 2(1), 89-100. https://doi.org/10.1080/1350462960020108

Ferrari, E., Ballegeer, A. M., Fuertes, M. A., Herrero, P., Delgado, L., Corrochano, D., Andrés-Sánchez, S., Marc, K., García-Vinuesa, A., Meira, P., Martinez, F. \& Ruiz, C. (2019). Improvement on social representation of climate change through a knowledge-based MOOC in spanish. Sustainability, 11(22), 6317. https://doi.org/10.3390/su11226317

Fischhoff, B. (2007). Non-persuasive communication about matters of greatest urgency: Climate change. Environmental Science \& Technology, (41), 72047208. https://doi.org/10.1021/es0726411

García-Rodeja, I., y Lima de Oliveira, G. (2012). Sobre el cambio climático y el cambio de los modelos de pensamiento de los alumnos. Enseñanza de las Ciencias, 30(3), 0195-218. https://raco.cat/index.php/Ensenanza/article/view/285690 
Gómez-Zwiep, S. (2008). Elementary teachers' understanding of students' science misconceptions: Implications for practice and teacher education. Journal of Science Teacher Education, 19(5), 437-454. https://doi.org/10.1007/s10972008-9102-y

González-Gaudiano, E. J., \& Meira-Cartea, P. Á. (2020). Educación para el cambio climático: ¿educar sobre el clima o para el cambio? Perfiles Educativos, 42(168), 157-174. https://doi.org/10.22201/iisue.24486167e.2020.168.59464

Heras, F. H., Meira-Cartea, P., \& Justel, A. (2017). La percepción social de los riesgos del cambio climático sobre la salud en España. Revista de Salud Ambiental, 17(1), 40-46. https://ojs.diffundit.com/index.php/rsa/article/view/842/802

Herrero, P., Andrés, S., Asensio, M. I., Ballegeer, A-M., Corrochano, D., Delgado, L., Izquierdo-Álvarez, V., Lagüela, S., Sampedro, J. M., \& Ruiz, C. (2018). A MOOC on the Science of Climate Change for primary and secondary teachers in Spanish. In Proceedings of the 6th International Conference on Technological Ecosystems for Enhancing Multiculturality (TEEM 2018) ACM, New York, USA. https://www.researchgate.net/publication/328972146_A_MOOC_on_the_Scie nce_of_Climate_Change_for_primary_and_secondary_teachers_in_Spanish

Higde, E., Oztekin, C., \& Sahin, E. (2017). Turkish pre-service science teachers' awareness, beliefs, values, and behaviours pertinent to climate change. International Research in Geographical and Environmental Education, 26(3), 253-263. https://doi.org/10.1080/10382046.2017.1330040

Intergovernmental Panel on Climate Change, IPCC (2021). Climate Change 2021: The Physical Science Basis. Contribution of Working Group I to the Sixth Assessment Report of the Intergovernmental Panel on Climate Change [Masson-Delmotte, V., P. Zhai, A. Pirani, S. L. Connors, C. Péan, S. Berger, N. Caud, Y. Chen, L. Goldfarb, M. I. Gomis, M. Huang, K. Leitzell, E. Lonnoy, J. B. R. Matthews, T. K. Maycock, T. Waterfield, O. Yelekçi, R. Yu, \& B. Zhou (eds.)]. Cambridge University Press. https://www.ipcc.ch/report/sixth-assessment-report-working-group-i/

Ikonomidis, S., Papanastasiou, D., Melas, D., \& Avgoloupis, S. (2012). The anthropogenic 'greenhouse effect': Greek prospective primary teachers' ideas about causes, consequences and cures. Journal of Science Education and Technology, 21(6), 768-779. https://www.learntechlib.org/p/167625/

Khalid, T. (2001). Pre-service teachers' misconceptions regarding three environmental issues. Canadian Journal of Environmental Education (CJEE), 6(1), 102-120. https://cjee.lakeheadu.ca/article/viewFile/290/198

Kikas, E. (2004). Teachers' conceptions and misconceptions concerning three natural phenomena. Journal of Research in Science Teaching: The Official Journal of the National Association for Research in Science Teaching, 41(5), 432-448. https://doi.org/10.1002/tea.20012

Leiserowitz, A. (2006). Climate change risk perception and policy preferences. Climatic Change, (77), 45-72. https://doi.org/10.1007/s10584-006-9059-9

Lopera Pérez, M., \& Villagrá Sobrino, S. (2020). Alfabetización climática en la formación inicial y continua de docentes. Uni-Pluriversidad, 20(1), 80-99. https://doi.org/10.17533/udea.unipluri.20.1.05 
Lorenzoni, I., Nicholson-Cole, S., \& Whitmarsh, L. (2007). Barriers perceived to engaging with climate change among the UK public and their policy implications. Global environmental change, 17(3-4), 445-459. https://doi.org/10.1016/j.gloenvcha.2007.01.004

Meira-Cartea, P. Á., Arto, M., Heras, F., Iglesias, L., Lorenzo, J. J., \& Motero, P. (2013). La respuesta de la sociedad española ante el cambio climático. Fundación Mapfre.

Meira-Cartea, P. A., Gutiérrez-Pérez, J., Arto-Blanco, M., \& Escoz-Roldán, A. (2018) Influence of academic education vs. common culture on the climate literacy of university students/Formación académica frente a cultura común en la alfabetización climática de estudiantes universitarios. Psyecology, 9(3), 301340. https://doi.org/10.1080/21711976.2018.1483569

Mochizuki, Y., \& Bryan, A. (2015). Climate change education in the context of education for sustainable development: Rationale and principles. Journal of Education for Sustainable Development, 9(1), 33-53. https://doi.org/10.1177/0973408215569109

Morote, A. F., Campo, B., \& Colomer, J. C. (2021). Percepción del cambio climático en alumnado de $4^{\circ}$ del Grado en Educación Primaria (Universidad de Valencia, España) a partir de la información de los medios de comunicación. Revista Electrónica Interuniversitaria de Formación del Profesorado, 24(1), 131-144. https://hdl.handle.net/11162/213111

Ojala, M. (2012). Hope and climate change: The importance of hope for environmental engagement among young people. Environmental Education Research, 18(5), 625-642. https://doi.org/10.1080/13504622.2011.637157

Ojala, M. (2015). Hope in the face of climate change: Associations with environmental engagement and student perceptions of teachers' emotion communication style and future orientation. The Journal of Environmental Education, 46(3), 133-148. https://doi.org/10.1080/00958964.2015.1021662

ONU (2015). Transformar nuestro mundo: la Agenda 2030 para el desarrollo sostenible. A/69/L85. http://www.un.org/ga/search/view_doc.asp? symbol=A/70/L.1\&Lang=S

Papadimitriou, V. (2004). Prospective primary teachers' understanding of climate change, greenhouse effect, and ozone layer depletion. Journal of Science $\begin{array}{llll}\text { Education } \quad \text { and 299-307. } & \text { 13(2), }\end{array}$ https://www.jstor.org/stable/40188791

Pérez-Rodríguez, U., Varela-Losada, M., Lorenzo-Rial, M. A., \& Vega-Marcote, P. (2017). Tendencias actitudinales del profesorado en formación hacia una educación ambiental transformadora. Revista de Psicodidáctica, 22(1), 60-68. https://doi.org/10.1387/RevPsicodidact.15608

Peters, A., \& Schneider, A. (2021). Cardiovascular risks of climate change. Nature Reviews Cardiology, (18)1-2. https://doi.org/10.1038/s41569-020-00473-5

Plein, C. (2019). Resilience, adaptation, and inertia: Lessons from disaster recovery in a time of climate change. Social Science Quarterly, 100(7), 2530-2541. https://doi.org/10.1111/ssqu.12658 
Puk, T. G., \& Stibbards, A. (2012). Systemic ecological illiteracy? Shedding light on meaning as an act of thought in higher learning. Environmental Education Research, 18(3), 353-373. https://doi.org/10.1080/13504622.2011.622840

Rosenthal, J. A. (2012). Statistics and Data Interpretation for Social Work (1st ed.). Springer Publishing Company.

Roser-Renouf, C., Maibach, E. W., Leiserowitz, A., \& Zhao, X. (2014). The genesis of climate change activism: From key beliefs to political action. Climatic change, 125(2), 163-178. https://doi.org/10.1007/s10584-014-1173-5

Scannell, L., \& Gifford, R. (2013). Personally relevant climate change: The role of place attachment and local versus global message framing in engagement.

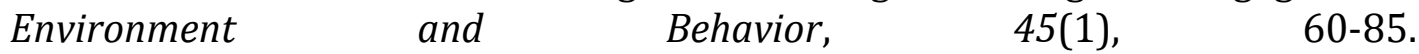
https://doi.org/10.1177/0013916511421196

Sharma, A. (2012). Global climate change: What has science education got to do with it? Science \& Education, 21(1), 33-53. https://doi.org/10.1007/s11191-0119372-1

Semenza, J. C., Hall, D. E., Wilson, D. J., Bontempo, B. D., Sailor, D. J., \& George, L. A. (2008). Public perception of climate change: voluntary mitigation and barriers to behavior change. American journal of preventive medicine, 35(5), 479-487. https://doi.org/10.1016/j.amepre.2008.08.020

Stevenson, R.B., Nicholls, J., y Whitehouse, H. (2017). What is climate change education? Curriculum Perspectives, 37(1), 67-71. https://doi.org/10.4018/978-1-7998-7512-3.ch013

Stevenson, K. T., Peterson, M. N., \& Bradshaw, A. (2016). How climate change beliefs among US teachers do and do not translate to students. PloS one, 11(9), e0161462. https://doi.org/10.1371/journal.pone.0161462

Tavares, A. O., Areia, N. P., Mellett, S., James, J., Intrigliolo, D. S., Couldrick, L. B., \& Berthoumieu, J. F. (2020). The European media portrayal of climate change: implications for the social mobilization towards climate action. Sustainability, 12(20), 8300. https://doi.org/10.3390/su12208300

Tobler, C., Visschers, V. H., y Siegrist, M. (2012). Consumers' knowledge about climate change. Climatic change, 114(2), 189-209. https://doi.org/10.1007/s10584011-0393-1

United Nations Development Programme, UNDP (2021). Peoples' climate vote. United Nations Development Programme and University of Oxford. https://www.undp.org/publications/peoples-climate-vote

Varela-Losada, M., Arias-Correa, A., \& Vega-Marcote, P. (2018). Training teachers committed to climate change mitigation. In Climate literacy and innovations in climate change education (pp. 307-321). Springer, Cham.

Witte, K., \& Allen, M. (2000). A Meta-analysis of Fear Appeals: Implications for Effective Public Health Campaigns. Health Educ. Behav.,(27), 591-615. https://doi.org/10.1177/109019810002700506 